\title{
State of the Art Hemorrhoidal Stapler Using a Modified Technique: A Step towards the Goals of Implement
}

\author{
George Anthimidis, Nikolaos Varsamis, Eleni Georgakoudi*, Epameinondas Fahantidis, \\ Kostas Ioannidis, George Basdanis \\ Interbalkan European Medical Center, Thessaloniki, Greece \\ Email: "helengeorgakoudi@gmail.com
}

Received 29 September 2015; accepted 9 November 2015; published 12 November 2015

Copyright (C) 2015 by authors and Scientific Research Publishing Inc.

This work is licensed under the Creative Commons Attribution International License (CC BY). http://creativecommons.org/licenses/by/4.0/

(c) () Open Access

\section{Abstract}

Background: Procedure for prolapse and hemorrhoids (PPH) has emerged as an alternative surgical treatment of symptomatic hemorrhoids. The operative technique along with the device to be used is constantly evolving. Aim: The aim of the present study was to evaluate results of PPH using a modified technique and an innovative circular stapler. Material and Methods: A prospective, comparative study of patients undergoing PPH for symptomatic Grade II and Grade III internal hemorrhoids with either $32 \mathrm{~mm}$ or $34 \mathrm{~mm}$ stapler diameter (Circular Stapler for HemorrhoidsCSH 32/34) during a 36 month period (1/1/2012-1/1/2015) was performed. A modified operative technique using two purse string sutures was employed. Patients were evaluated and compared in terms of postoperative complications, operation time, length of hospital stay and time to return to normal activities. Results: A total of 100 patients were included: 50 in the CSH 32 group and 50 in the CSH 34 group. Complication rates were equal for both stapler diameters ( $32 \mathrm{~mm}$ and $34 \mathrm{~mm}$ ). Serious or life threatening complications were not encountered and need for reintervention was never met. Operation time, length of hospital stay and time to return to normal activities were similar in both groups. Conclusions: Our study suggests that there are no significant differences between the two available diameters of a new-fangled circular stapler, using a modified technique. It seems that progress of hemorrhoidal staplers has led to a step towards the goals of implement and from then on complications of surgical practice could be merely a matter of operative technique.

\section{Keywords}

Hemorroids, Stapler, Procedure for Prolapse and Hemorrhoids

\footnotetext{
${ }^{*}$ Corresponding author.
} 


\section{Introduction}

A number of surgical procedures have been described for elective treatment of symptomatic hemorrhoids. Procedure for prolapse and hemorrhoids (PPH) has emerged as an alternative surgical approach, associated with less postoperative pain and disability. Yet, serious adverse events and critics have been reported [1]. The operative technique along with the material to be used is constantly evolving. The aim of the present study is to evaluate results of PPH using a modified technique and an innovative circular stapler.

\section{Material and Methods}

A prospective, comparative study of patients undergoing PPH with either $32 \mathrm{~mm}$ or $34 \mathrm{~mm}$ stapler diameter (EVOMED Disposable Circular Stapler for Hemorrhoids-CSH 32/34) during a 36 month period (1/1/20121/1/2015) was performed. All patients who underwent PPH for symptomatic Grade II and Grade III internal hemorrhoids and partial thickness internal rectal prolapse were included. Exclusion criteria were patients who were operated for Grade IV internal and combined internal-external hemorrhoids hemorrhoids. Patients were stratified according to age, sex, grade of internal hemorrhoids, and were randomly allocated to PPH with stapler diameter $32 \mathrm{~mm}$ or $34 \mathrm{~mm}$ by the same surgeon. All patients received subcutaneous heparin; enema and antibiotic prophylaxis were given according to the surgeon's usual routine. Postoperative follow-up was performed at 15 and 30 days (early), and 3 months (late). The patients were assessed by two "blinded" observers (surgeons). All data were recorded using Statistical Package for the Social Sciences (SPSS) 21 for Windows for statistical analyses. As the main objective of the present study was to evaluate patients undergoing PPH with either $32 \mathrm{~mm}$ or $34 \mathrm{~mm}$ stapler diameter, data were tabulated comparing between these two groups of patients in terms of rectal bleeding, urinary retention, need for postoperative analgesics (early postoperative pain and chronic pain), fecal urgency, tenesmus, fecal incontinence, stenosis, thrombosed external piles, anal fissure, fecal impaction, proctitis, local abscess and fistula (including rectovaginal fistula), pelvic sepsis, rectal hematoma leading to bowel obstruction, skin tags, stricture, complete rectal obliteration, rectal pocket, rectal dysplasia/adenocarcinoma, penile trauma after active anal intercourse, retropneumoperitoneum, pneumomediastinum, recurrence, and need for reoperation. Rectal bleeding was defined as a complication when bleeding requiring hospital re-admission and/or surgical intervention was encountered. The assessment of postoperative pain as a complication was based on need for analgesics more than $4 \mathrm{~g}$ of paracetamol/24 h.

\section{Operative Technique}

General anesthesia was preferable and the patient was placed in an exaggerated dorsal lithotomy position, with the buttocks extending beyond the edge of the table and the legs held in stirrups. The anal verge was gently massaged and dilated, digital rectal examination was performed and the obturator was inserted alone as part of the dilation process in order to prevent damage to the internal sphincter. Then the obturator was removed and the circular anal dilator, along with the obturator in place, was introduced, pushing the mucosal prolapse back into the anal canal. Care was taken to guarantee the retractor covers the dentate line completely and circumferentially before securing it in place, in order to avoid damage to the dentate line and the internal sphincter. The retractor was secured with perianal stay sutures. The obturator was replaced with the fenestrated obturator to facilitate placement of two separate purse string sutures, each at 4 and $5 \mathrm{~cm}$ from the dentate line.

Once the fenestrated obturator was in appropriate position, the first purse string suture was placed at $5 \mathrm{~cm}$ from the dentate line, with mucosal-submucosal bites, using 2 - 0 Prolene suture on a UR-6 needle, beginning at 3 o'clock and progressing close together (6 - 12 small bites). Care was taken to remove and replace the obturator rather than simply turn it clockwise. This avoids pulling the mucosa and creates a more complete purse string circumferentially. The purse string should lie at the same level circumferentially.

A second stitch was then placed at $4 \mathrm{~cm}$ from the dentate line to assure the purse string is secure and even circumferentially. The second stitch began at 6 o'clock and progressed in quadrants. The fenestrated obturator was removed and replaced with the fully open Circular Stapler for Hemorrhoids-CSH 32/34.

The first purse string suture $(5 \mathrm{~cm})$ was sutured around the shaft of the anvil and cut, and the two suture ends of the second purse string suture $(4 \mathrm{~cm})$ were brought out through the eyelets of the stapler using the hook and secured externally with a surgeon's knot.

It should be confirmed that the entire anvil is proximal to the purse string sutures and that the complete cir- 
cumference of the mucosa is up against the center of the rod.

While maintaining moderate distal traction on the suture, the stapler was being closed by allowing the stapler to enter the anus rather than pulling the mucosa to the stapler. Thus the prolapsed mucosa began to be drawn into the stapler casing.

Care was taken so that the stapler was aligned along the axis of the anal canal. The stapler was then closed to the end by full rotation of the knob of the instrument clockwise. The red indicator should be positioned at the center of the green window of the handle at this time and held in position for $30 \mathrm{~s}$. If the patient was female, the posterior vaginal wall was manually palpated to avoid incorporating it in the staple line. It is important during these $30 \mathrm{~s}$ the patient does not Valsalva to avoid avulsing the mucosa.

The safety was then released and the stapler was subsequently fired and held in position for $30 \mathrm{~s}$ to act as a tamponade and enhance hemostasis. At this point, the stapler handle was released and turned one-half to three-quarters of a full turn to partially open the anvil. Additional turns may lead to interposition of mucosa between the anvil head and the upper edge of the circular anal dilator. The stapler was then removed from the anus.

The anvil was opened and the rectal mucosa-submucosa cut to pass it off the table for pathologic evaluation. Inspection of the specimen was carried out to confirm that the technique has been appropriately performed. It should contain a $3 \mathrm{~cm}$-wide strip of rectal mucosa and possibly hemorrhoid tissue, with no or minimal muscle. The fenestrated obturator was replaced in the anal canal. Proper placement of the staple line was at least $2 \mathrm{~cm}$ to $4 \mathrm{~cm}$ from the dentate line. Any bleeding from the staple line was reinforced with figure of 8 absorbable sutures (2.0 Vicryl ${ }^{\circledR}$ ) until hemostasis was considered to be acceptable. The perianal stay sutures were cut and all retractors removed. A foam-like product was inserted into the anal canal to enhance hemostasis.

The wound was dressed with $4 \times 4$ gauze and a peripad.

\section{Results}

A total of 100 patients were included: 50 in the EVOMED Disposable Circular Stapler for Hemorrhoids-CSH 32 group and 50 in the EVOMED Disposable Circular Stapler for Hemorrhoids-CSH 34 group.

Descriptive statistics were compiled to characterize the study population and to examine group differences. Continuous/discrete variables emerged without normal distribution and they were described by median value and range (minimum-maximal value).

Statistical comparisons were performed using the Chi-square test for independence for the categorical dependent variables and the Mann-Whitney $\mathrm{U}$ test for the continuous dependent variables. P values below 0.05 were considered significant.

Comparison of groups for certain categorical variables that did violate the assumption of the Chi-square Test concerning the "minimum cell frequency" (at least $80 \%$ of cells with expected frequencies of 5 or more) was carried out using Fisher's Exact Probability Test.

The comparability of the two groups is shown in Table 1.

The parameters were well matched. There was no statistically significant heterogeneity among the patient characteristics and stage of disease.

Operation time, length of hospital stay and time to return to normal activities were similar in both groups

Table 1. Patient characteristics and stage of disease.

\begin{tabular}{ccc}
\hline & CSH $32 \mathrm{n}=50$ & CSH $34 \mathrm{n}=50$ \\
\hline $\begin{array}{c}\text { Patient characteristics } \\
\text { Age: median (minimum-maximum) }\end{array}$ & $50(35-62)$ & $50(35-60)$ \\
Sex ration & $33: 17$ & $33: 17$ \\
Male:female & & 25 \\
Disease stage & 25 & 25 \\
II & 25 & \\
III & & \\
\hline
\end{tabular}


(Table 2).

The type, number and percentage of complications along with the statistical results are illustrated in Table 3. Complications did not occur in $72 \%$ of all patients.

Interestingly, numerous well established complications were not noticed. Specifically, fecal incontinence, stenosis, thrombosed external piles, local abscess and fistula (including rectovaginal fistula), pelvic sepsis, rectal hematoma leading to bowel obstruction, stricture, complete rectal obliteration, rectal pocket, rectal dysplasia/ adenocarcinoma, penile trauma after active anal intercourse, retropneumoperitoneum, pneumomediastinum were not reported in both groups. Moreover need for reoperation was never encountered.

Complication rates were similar for both stapler diameters (32 $\mathrm{mm}$ and $34 \mathrm{~mm}$ ).

No differences were found in any of the follow-up assessments in any of the variables analyzed.

\section{Discussion}

Hemorrhoids are cushions of submucosal tissue that contain arterioles, venules, and smooth-muscle fibers located in the anal canal. Hemorrhoids are considered to be part of the continence mechanism. Increased abdominal pressure, excessive straining and hard stools increase venous engorgement of the hemorrhoidal plexus and may lead to symptomatic hemorrhoidal prolaps, bleeding, and thrombosis.

External hemorrhoids are located distal to the dentate line. They are covered by the richly innervated anoderm and thrombosis of an external hemorrhoid may cause considerable pain. Moreover, they may produce itching and difficulty with hygiene if they are large. Treatment of external hemorrhoids is only indicated for symptomatic relief.

Internal hemorrhoids are located proximal to the dentate line. They may prolapse or bleed, but rarely become painful, since they are covered by insensate anorectal mucosa. However they may become painful, if they develop thrombosis and necrosis, typically owing to severe prolapse, incarceration, and/or strangulation. Internal hemorrhoids are graded in accordance with the extent of prolapse. First-degree hemorrhoids bulge into the anal canal and may prolapse beyond the dentate line on straining. Second-degree hemorrhoids prolapse through the anus but reduce spontaneously. Third-degree hemorrhoids prolapse through the anal canal but need manual reduction. Fourth-degree hemorrhoids prolapse but cannot be reduced and are susceptible to strangulation.

Combined internal and external hemorrhoids straddle the dentate line and have features of both internal and external hemorrhoids.

Worldwide, the prevalence of symptomatic hemorrhoids is estimated to be $4.4 \%$ in the general population. The prevalence of hemorrhoids increases with age, with a peak in persons between 45 - 65 years.

Table 2. Time parameters.

\begin{tabular}{|c|c|c|c|}
\hline & $\mathrm{CSH} 32 \mathrm{n}=50$ & $\mathrm{CSH} 34 \mathrm{n}=50$ & \multirow{2}{*}{$\mathrm{p}^{*}$} \\
\hline \multicolumn{3}{|c|}{ Median (minimum-maximum) } & \\
\hline Operation time (min) & $30(20-45)$ & $30(20-45)$ & $0.648(>0.05)$ \\
\hline Length of hospital stay & $1(1-2)$ & $1(1-2)$ & $1.000(>0.05)$ \\
\hline Time to return to normal activities & $8(7-10)$ & $8(7-10)$ & $0.826(>0.05)$ \\
\hline
\end{tabular}

Table 3. Type, number and percentage of complications.

\begin{tabular}{cccc}
\hline & CSH $32 \mathrm{n}=50$ & CSH $34 \mathrm{n}=50$ & $\mathrm{p}^{*}$ \\
\hline Rectal bleeding & $\mathrm{n}=3(6 \%)$ & $\mathrm{n}=3(6 \%)$ & $1.000(>0.05)$ \\
Urinary retention & $\mathrm{n}=2(4 \%)$ & $\mathrm{n}=2(4 \%)$ & $1.000(>0.05)$ \\
Early postoperative pain & $\mathrm{n}=2(4 \%)$ & $\mathrm{n}=3(6 \%)$ & $1.000(>0.05)$ \\
Constipation & $\mathrm{n}=3(6 \%)$ & $\mathrm{n}=3(6 \%)$ & $1.000(>0.05)$ \\
Chronic pain & $\mathrm{n}=3(6 \%)$ & $\mathrm{n}=1(2 \%)$ & $1.000(>0.05)$ \\
Recurrence & $\mathrm{n}=1(2 \%)$ & $\mathrm{n}=5(10 \%)$ & $1.000(>0.05)$ \\
Residual skin tags & $\mathrm{n}=4(8 \%)$ & & $1.000(>0.05)$
\end{tabular}


Treating internal hemorrhoids by surgically reducing the anal mucosal prolapse using a circular stapler was conceived by Dr. Antonio Longo to minimize postoperative discomfort. The procedure for prolapse and hemorrhoids (PPH) is carried out with a specially designed stapling device to excise a complete ring of redundant mucosa or expanded internal hemorrhoids above the dentate line. The key feature of this method is the lack of any apparent perianal wounds, which therefore should be less painful than conventional hemorrhoidectomy [2].

Yet, serious adverse events and critics have been reported about PPH [1]. The operative procedure together with the material to be used is continuously evolving. The aim of the present study is to evaluate results of PPH using a modified technique and an innovative circular stapler.

As far as we could elicit from the literature, this clinical trial is the first to evaluate the effectiveness of a specifically designed hemorrhoidal circular stapler available at $32 \mathrm{~mm}$ and $34 \mathrm{~mm}$ diameter (EVOMED Disposable Circular Stapler for Hemorrhoids-CSH 32/34) for PPH with a standard modified technique. The uniform effect of our technique was reflected in the $3 \mathrm{~cm}$-wide strip of rectal mucosa and possibly hemorrhoid tissue, with no or minimal muscle. As the main objective of the present study was to evaluate patients undergoing PPH with either $32 \mathrm{~mm}$ or $34 \mathrm{~mm}$ stapler diameter, data were tabulated comparing between these two groups of patients. The two groups were well matched for the patient characteristics and stage of disease. Patient assessment was "double-blind" as neither the examiner nor the patient knew which diameter (32 mm or $34 \mathrm{~mm}$ ) of the hemorrhoidal circular stapler had been used.

Differences in terms of operation time, length of hospital stay and time to return to normal activities were not found and our results appear to concur with previous reports [3].

Complication rates were similar for both groups. From the results of our statistics, we can easily find that there are no differences between CSH 32 and 34. Our results appear to be quite acceptable in accordance with systematic reviews and meta-analyses [4] [5].

Complications did not occur in $72 \%$ of all patients, while one multicenter study reported that $36.4 \%$ of patients had at least one adverse event after PPH [6]. The only complications that occurred in our series were: rectal bleeding, urinary retention, early postoperative pain, chronic proctalgia, constipation, recurrence of prolapsed and residual skin tags. However, none of these complications exceeded $10 \%$.

Additionally, although well established adverse events have been reported in the past, it was interesting to discover in the present study that unusual, serious or life threatening complications were not encountered and need for reintervention was never met [7].

The fine results may be related to the unique physical characteristics and properties of EVOMED Disposable Circular Stapler for Hemorrhoids, which is available at both $32 \mathrm{~mm}$ and $34 \mathrm{~mm}$ stapler diameter, and applies two staggered rows of 32 and 34 titanium staples respectively, by a staple leg length of $3.8 \mathrm{~mm}$. Additionally, the satisfactory results may be due to our modified technique utilizing two purse string sutures, which appears to ensure a consistent $3 \mathrm{~cm}$-wide strip of rectal mucosa and possibly hemorrhoid tissue. By means of our modification, the second purse string suture $(4 \mathrm{~cm})$ is not sutured around the shaft of the anvil and the two suture ends are brought out through the eyelets of the stapler using the hook and secured externally with a surgeon's knot.

Nevertheless, the present study is subject to certain limitations. The sample size is not large, and the postoperative follow-up was not performed later than 3 months.

\section{Conclusion}

In conclusion, the selection of a specific circular stapler may be based on scientific data, as well as on the preferences that one has learned from mentors and/or during training. Comprehension of the individual characteristics of available staplers is important to make an educated selection. Up to now, no one device is ideal and compromises have to be made. Our study suggests that EVOMED Disposable Circular Stapler for Hemorrhoids-CSH 32/34 may be a step towards the goals of implement and from then on complications of surgical practice could be merely a matter of operative technique. Since there are no significant differences between these two instruments, using our modified technique, additional studies may be conducted to test the consistency of our findings in larger sample sizes and expectantly in other study populations.

\section{Approval}

This study was approved by the patients themselves. 


\section{References}

[1] Porrett, L.J., Porrett, J.K. and Ho, Y.-H. (2015) Documented Complications of Staple Hemorrhoidopexy: A Systematic Review. International Surgery, 100, 44-57. http://dx.doi.org/10.9738/INTSURG-D-13-00173.1

[2] Longo, A. (1998) Treatment of Haemorrhoid Disease by Reduction in Mucosal and Haemorrhoidal Products with a Circular Stapling Device-New Procedure. Proceedings of the 6th World Congress of Endoscopic Surgery, Rome.

[3] Chen, J.S. and You, J.F. (2010) Current Status of Surgical Treatment for Hemorrhoids—Systematic Review and Meta-Analysis. Chang Gung Medical Journal, 33, 488-500.

[4] Nisar, P.J., Acheson, A.G., Neal, K. and Scholefield, J.H. (2004) Stapled Haemorrhoidopexy Compared with Conventional Haemorrhoidectomy: Systematic Review of Randomized Controlled Trials. Diseases of the Colon \& Rectum, 47, 1837-1845. http://dx.doi.org/10.1007/s10350-004-0679-8

[5] Jayaraman, S., Colquhoun, P.H. and Malthaner, R.A. (2007) Stapled Haemorrhoidopexy Is Associated with a Higher Long-Term Recurrence Rate of Internal Hemorrhoids Compared with Conventional Excisional Hemorrhoidal Surgery. Diseases of the Colon \& Rectum, 50, 1297-1305. http://dx.doi.org/10.1007/s10350-007-0308-4

[6] Senagore, A.J., Singer, M. and Abcarian, H. (2004) A Prospective, Randomized, Controlled Multicenter Trial Comparing Stapled Haemorrhoidopexy and Ferguson Hemorrhoidopexy: Perioperative and One-Year Results. Diseases of the Colon \& Rectum, 47, 1824-1836. http://dx.doi.org/10.1007/s10350-004-0694-9

[7] Pescatori, M. and Gagliardi, G. (2008) Postoperative Complications after Procedure for Prolapsed Hemorrhoids (PPH) and Stapled Transanal Rectal Resection (STARR) Procedures. Techniques in Coloproctology, 12, 7-19.

http://dx.doi.org/10.1007/s10151-008-0391-0 